www.jmscr.igmpublication.org

Index Copernicus Value: 79.54

ISSN (e)-2347-176x ISSN (p) 2455-0450

crossref DOI: https://dx.doi.org/10.18535/jmscr/v7i5.163

\title{
Recurrent Diffuse Alveolar Haemorrhage in a Patient with IgA Nephropathy Related End Stage Kidney Disease: A Rare Complication
}

\author{
Author \\ Vishal Singh \\ Division of Nephrology, Department of Medicine \\ 7 AF Hospital, Kanpur Cantt (UP), India
}

\begin{abstract}
Immunoglobulin A nephropathy (IgAN) is the commonest glomerulopathy worldwide. It has a wide spectrum of clinical presentation, ranging from isolated hematuria to rapidly progressive glomerulonephritis. Diffuse alveolar haemorrhage $(\mathrm{DAH})$ is a life-threatening medical emergency and commonly presents with hemoptysis, anemia, hypoxemia and diffuse alveolar infiltrates. The most common cause for DAH is pulmonary capillaritis secondary to systemic autoimmune disorders. Association of DAH with IgAN is uncommon and it often goes undiagnosed. This is because the symptoms mimic pneumonia and fluid overload, which are much more commonly encountered in patients with end stage kidney disease. The diagnosis requires early bronchoscopy and bronchoalveolar lavage. A high index of clinical suspicion, early recognition and prompt treatment is the key to reduce mortality. Here we present a 21-year-old male who presented one year back with fluid overload and advanced azotemia. The kidney biopsy showed IgA nephropathy with significant chronicity. He was started on hemodialysis; 3 months in to hemodialysis, he developed recurrent episodes of treatment unresponsive DAH. The response to steroids were poor. The subsequent response to plasmapheresis and anti-CD 20 monoclonal antibodies was suboptimal and he finally responded to a combination of mycophenolate mofetil and oral steroids.

Keywords: IgA nephropathy, diffuse alveolar haemorrhage, pulmonary renal syndrome.

\section{Introduction}

IgAN is a mesangioproliferative glomerulonephritis characterized by diffuse mesangial deposition of immunoglobulin A ( $\operatorname{Ig} \mathrm{A})$. It is the most common primary glomerulopathy worldwide. The spectrum of clinical presentation is wide and varies from asymptomatic hematuria to rapidly progressive renal failure. The commonly encountered scenario in clinical practise is a gradual but progressive decline in kidney function culminating in end stage kidney disease.

\section{$\mathrm{DAH}$ is a clinico-pathological syndrome} characterized by bleeding into the alveolar space. This bleeding is a consequence of disruption of the alveolar-capillary basement membrane secondary to injury or inflammation of the arterioles, venules or the capillaries. The onset is generally abrupt and presentation includes cough, haemoptysis, fever and dyspnoea; with investigations showing a drop-in haemoglobin and appearance of new alveolar infiltrate on the chest X-ray. The causes for DAH are many and the management includes supportive care along with treatment of the underlying etiology.
\end{abstract}


DAH and glomerulonephritis can co-occur in many conditions and are clubbed under a common syndrome called 'Pulmonary renal syndrome' (PRS). Some of the common causes of PRS include anti glomerular basement membrane (anti GBM) disease, systemic lupus erythematosus (SLE), antineutrophilic cytoplasmic antibody (ANCA) associated vasculitis, Henoch-Schoenlein purpura (HSP), drugs and acute respiratory distress syndrome. Association of DAH with IgAN is rare, with occasional case reports and case series being described in the literature. We present a young male with IgAN related end stage kidney disease who developed recurrent episodes of DAH while on hemodialysis. We intend to discuss the challenges faced during the management along with the review of existing literature.

\section{Case Report}

A 21 years old male with no known co-morbidities, sought medical consult one year back, for progressively worsening pitting pedal oedema, breathlessness and loss of appetite. He had no arthralgia, abdominal pain or skin rash. He denied history of passage of blood or stone in the urine, frequent urinary tract infection, double voiding, analgesic abuse, consumption of alternative medicines, oral ulcers, or preceding fever. Examination showed pallor, elevated blood pressure (180/ $110 \mathrm{~mm} \mathrm{Hg}$ ) and pedal oedema. The examination of cardiovascular and respiratory systems was normal. Laboratory findings are as follows: hemoglobin: 6.1 grams/ decilitre (microcytic, hypochromic red blood cells), total leukocyte count: 7900/ cubic millimetre, Platelets: $1.44 \times 10^{3} /$ cubic millilitre, blood urea nitrogen: 250 milligram/ decilitre, serum creatinine: 22.5 milligram/ decilitre, total protein: 5.6 grams/ decilitre, serum albumin: $2.6 \mathrm{gram} /$ decilitre. The urine analysis showed $3+$ proteins and 10-12 dysmorphic red blood cells per high power field. The urine protein to creatinine ratio was 3.3 gram/ gram of creatinine. The serum electrolytes were normal and the arterial blood gas analysis showed partially compensated metabolic acidosis. The ultrasound showed normal sized echogenic kidneys with loss of cortico-medullary differentiation. The markers for anti GBM disease, ANCA associated disease and SLE was negative. The serum complements were normal. He was started on thrice weekly hemodialysis, and after the initial stabilization a kidney biopsy was done. The biopsy showed features of IgAN with significant chronicity. 6 out of 7 glomeruli were obsolescent and more than $50 \%$ of the sampled cortical area showed interstitial fibrosis/ tubular atrophy. Hemodialysis was continued along with supportive care and he was worked up for a live related kidney transplant. Three months into hemodialysis, he developed sudden onset fever, cough and shortness of breath. There was no hemoptysis or pleuritic type chest pain. Examination showed tachycardia, tachypnoea and bilateral crepitations involving the inter and infra-scapular region. Pending the investigation reports, with a provisional diagnosis of community acquired pneumonia; he was started on empiric intravenous antibiotics. Investigations showed a fall in hemoglobin from 10 to 9.2 grams/ decilitre, leucocytosis and a normal serum procalcitonin. The chest X-ray showed diffuse alveolar infiltrates involving bilateral middle and lower zones (Figure 1). A high-resolution computed tomography scan of the chest showed bilateral extensive area of ground glass opacities with dense consolidation (Figure 2). The blood and urine cultures were sterile; the sputum microscopy for gram stain, acid fast bacilli and fungus did not show any pathogen. He continued to worsen despite therapy and required non-invasive ventilation. An urgent bronchoscopy showed presence of ongoing bleed and the bronchoalveolar lavage showed haemorrhagic fluid (Figure 3). The transbronchial lung biopsy (TBLB) showed presence of pulmonary capillaritis. The TBLB specimen was not subjected to immunohistochemistry for $\operatorname{IgA}$ deposits. The serum IgA levels was not checked. With a diagnosis of $\mathrm{DAH}$, he was given three pulses of intravenous methyl-prednisolone (500 mg, each) followed by 1 milligram per kilogram of body weight per day, of oral prednisolone. The response however was 
suboptimal with no change in the clinical status. It was at this time that a need was felt for more aggressive immunosuppression for the immune mediated DAH. Seven sessions of plasmapheresis along with two doses of rituximab (375 milligram per meter square of body surface area) was given. Following the therapy his ventilatory requirements improved and over the next few weeks, he was able to do activities of daily living. The oral steroids were continued with a plan to slowly taper it over the next four-six months. One month following the initial episode of $\mathrm{DAH}$, he had a recurrence requiring non-invasive ventilation. The haemoglobin fell from 8.9 to 4.2 grams/ decilitre and he was transfused four bags of packed red blood cells. Intravenous methyl-prednisolone $(500 \mathrm{mg}$, each) was repeated, following which he showed improvement. In view of recurrent episodes of DAH, he was offered a cyclophosphamide-based therapy, but was unwilling. He again had a recurrence of $\mathrm{DAH}, 3$ weeks later and the haemoglobin fell to 4.8 grams/ decilitre. The markers for anti GBM disease, ANCA associated disease and SLE was repeated and was negative. The serum complements continued to be normal. The polymerase chain reaction for cytomegalovirus was negative. Recurrent life-threatening DAH, despite high dosages of steroids, rituximab and plasmapheresis prompted us to start him on oral mycophenolate mofetil. The subsequent course was largely uneventful. There was no recurrence of DAH. The dose of mycophenolate was reduced from 2 grams to 1 gram per day. After an observation of 6 months following the last episode of DAH he was taken up for a live related kidney transplantation with his mother as donor. The induction therapy was with rabbit antithymocyte globulin (3 milligram/ kilogram body weight) and the maintenance immunosuppression included tacrolimus, mycophenolate and prednisolone. The posttransplant course has been uneventful and he is maintaining a good graft function.

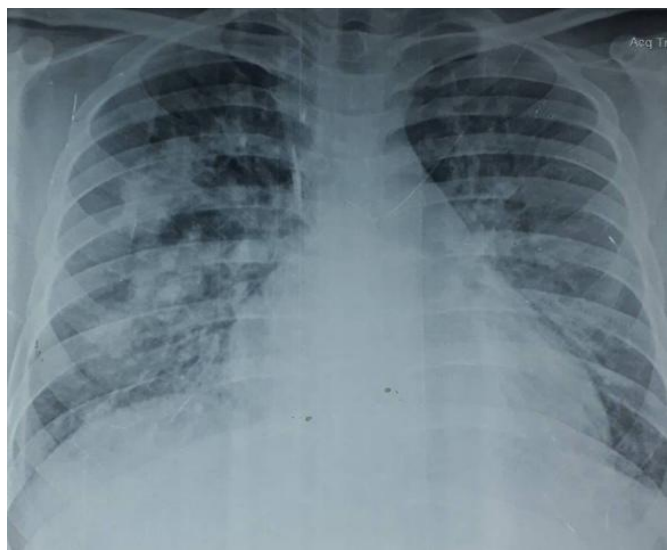

Figure 1: Chest X-ray PA view showing diffuse infiltrative opacification pattern with slight predilection towards the middle and lower zone and some apical sparing.

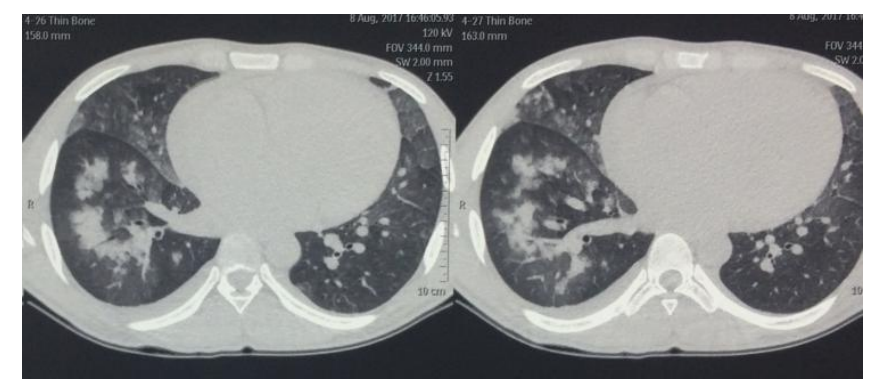

Figure 2: High resolution computed tomography of the chest (Lung window) showing lobar areas of ground glass opacities and consolidation.

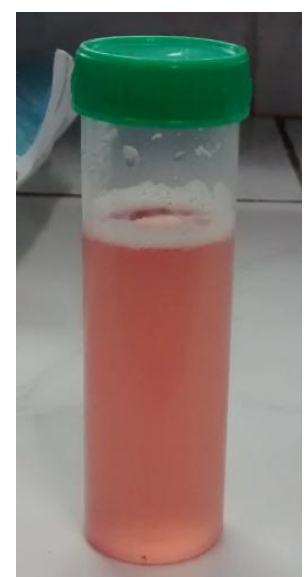

Figure 3: Bronchoalveolar lavage fluid showing haemorrhagic return fluid.

\section{Discussion}

IgAN also called 'Berger disease' is the most prevalent glomerular disease worldwide. It was first described by Jean Berger in 1968, when immunofluorescence technique was introduced for evaluation of renal biopsy. Unlike other glomerular 
diseases which are defined by the morphological features, $\operatorname{IgAN}$ is unique, in that, it is defined by the presence of mesangial $\operatorname{Ig} \mathrm{A}$. The morphology on the light microscopy is variable. The prevalence of IgAN varies with the geographical area and based on biopsy studies, it is estimated to be around 1.5 per 100,000 population $^{(1)}$. IgA is the most abundant immunoglobulin in the body and has two subclasses, $\operatorname{IgA} 1$ and $\operatorname{IgA} 2$. Mucosal antigen challenge causes the plasma cells of the mucosa associated lymphoid tissue to secrete polymeric $\operatorname{IgA}$, which crosses the epithelium into the mucosal fluid and is released as secretory $\operatorname{IgA}$. This secretory $\operatorname{IgA}$ is chiefly concerned with mucosal defence. The bone marrow derived circulating $\operatorname{IgA}$, which are mostly monomeric IgA1 have an unknown function. One third of patients with IgAN have elevated serum IgA levels; both polymeric and monomeric IgA1. The mesangial IgA deposits in IgAN are predominantly polymeric IgA1. The source of this $\operatorname{IgA}$ is believed to be from misplaced mucosal derived plasma cells following mucosal priming by upper respiratory or gastrointestinal mucosal antigen. The initiation of disease is attributed to a 'multi hit process'; the common thread being presence of circulating and glomerular immune complexes comprising of galactose-deficient IgA1, an $\mathrm{IgG}$ autoantibody directed against the hinge region O-glycans, and $\mathrm{C} 3$. This aberrantly glycosylated $\operatorname{IgA} 1$ is a heritable trait ${ }^{(2)}$. The immune complex, so formed is nephritogenic and is responsible for local inflammation and cell proliferation which in turn contributes to fibrosis and manifest as chronic kidney disease. The usual presentation of IgAN includes asymptomatic hematuria and or progressive renal disease. In some, it may also present with synpharyngitic macroscopic hematuria or nephrotic range proteinuria. IgAN presenting with DAH is a very rare, life threatening complication.

DAH is a clinicopathologic term characterized by bleeding into the alveolar spaces, i.e. accumulation of intra-alveolar red blood cells. It is a consequence of injury or inflammation to the small vessels, i.e. arterioles, venules and the capillaries and manifests as hemoptysis, anemia, diffuse radiographic pulmonary infiltrates, and or hypoxemic respiratory failure. Hemoptysis may be absent at the time of presentation in up to one-third of the patients ${ }^{(3)}$. The etiology for DAH can be broadly divided into pulmonary capillaritis (SLE, granulomatosis with polyangiitis, cryoglobulinemia etc.), DAH with bland pulmonary haemorrhage (coagulopathy, elevated left ventricular end diastolic pressure) and DAH secondary to diffuse alveolar damage (acute respiratory distress syndrome and drugs like nitrofurantoin, sirolimus and amiodarone). DAH is potentially life threatening and the natural course is unpredictable. The survival depends on the underlying etiology with maximum mortality being associated with those secondary to anti GBM antibody disease, granulomatosis with polyangiitis and SLE.

IgAN and HSP share close pathogenetic link despite differences in age of onset and natural history. While DAH is relatively common in HSP with a prevalence of $0-5 \%$, the same is not true for $\operatorname{IgAN}$. Lai et al were the first to describe a series of patients of pulmonary haemorrhage associated with $\operatorname{IgAN}^{(4)}$. Of the three patients described all died; two of respiratory failure, and one following cardiac arrest. Since then there had been isolated case reports. In a recent case report series, reported in the year 2017, the authors performed a literature search and were able to identify 23 such patients in 19 case reports worldwide ${ }^{(5)}$. The other significant pulmonary involvement noted by the authors included, interstitial lung disease and organizing pneumonia. They found that dyspnoea (84\%), hemoptysis (74\%), cough $(53 \%)$ and fever (47\%) were the most common presenting complaints. Our patient also presented with fever, dyspnoea and cough and we were later able to demonstrate hemoptysis on bronchoscopy. Further, the bronchoalveolar lavage and transbronchial lung biopsy confirmed the presence of DAH and small vessel vasculitis respectively. We excluded the other common causes of DAH during the initial serological evaluation of chronic kidney disease thus localizing the possible etiology to $\operatorname{IgAN}$. The 
precise mechanisms of extra renal bleed, i.e. DAH in IgAN is not known. It is hypothesized that the possible etiology is immune complex mediated damage to the pulmonary microvasculature basement membrane ${ }^{(6)}$. We also feel that while galactose deficient IgA1 related immune complex has a major role in immuno-pathogenesis of IgAN, the same circulating complex may produce extra renal manifestations like DAH. It possibly requires multiple secondary hits, which may possibly account for the rare occurrence of DAH in IgAN. It is also possible that the pulmonary involvement in $\mathrm{IgAN}$ is not so uncommon, but is either undiagnosed, unrecognized or subclinical.

Due to the rare nature of this syndrome, there is no evidence based therapeutic protocol. The standard therapeutic protocol for other autoimmune vasculitis consists of an initial intensive "remission induction" phase followed by a less intensive "maintenance" phase. In most of the published case reports of IgAN-DAH complex, steroids and immunosuppression have been used. Rajagopala $S$, et al in their case series observed that steroids with or without immunosuppression were used in $89 \%$ of the patients ${ }^{(5)}$. They also noted that the most common protocol used was steroids with cyclophosphamide as induction therapy, followed by methotrexate or azathioprine as maintenance immunosuppression. The other adjunct therapy used included rituximab, plasmapheresis, mechanical ventilation and dialysis. Therapeutic protocol of DAH in HSP, a similar condition, suggests combination therapy with corticosteroids and immunosuppressants may be the most effective ${ }^{(7)}$. In our case, the patient had an inadequate response to high dose steroid and based on the hypothesized pathophysiology he was given plasmapheresis and intravenous rituximab. He was young and unmarried and unwilling for cyclophosphamidebased therapy. What made our case unique and challenging, was an inadequate response to the therapy and three distinctly separate episodes of DAH occurring within a short course of time. He finally responded to a combination of mycophenolate with oral steroids and later underwent a successful live related kidney transplant.

\section{Conclusion}

$\mathrm{DAH}$ is a rare but serious complication of $\operatorname{IgAN}$ and is associated with significant morbidity and mortality. Diagnosis requires a high index of suspicion, as overt hemoptysis may be absent; this is especially so as the symptom complex often mimics other conditions like fluid overload and pneumonia. Bronchoscopy and bronchoalveolar lavage are indispensable tool in establishing the diagnosis in such patients. The exact cause for this "extra renal bleed" in IgAN is not known but it possibly represents IgA capillaritis. Though no evidence-based treatment protocol exists, the therapeutic options include steroids, plasmapheresis, cyclophosphamide and rituximab. Mycophenolate remains a good option, especially for the subset of patients who are either therapy resistant, treatment non-responsive, or having recurrent life-threatening DAH.

\section{Conflict of interest: None}

\section{References}

1. McGrogan A, Franssen CF, de Vries CS: The incidence of primary glomerulonephritis worldwide: A systematic review of the literature. Nephrol Dial Transplant 26: 414430, 2011.

2. Kiryluk K, Moldoveanu Z, Sanders JT, Eison TM, Suzuki H, Julian BA, Novak J, Gharavi AG, Wyatt RJ: Aberrant glycosylation of $\operatorname{IgA} 1$ is inherited in both pediatric IgA nephropathy and HenochSchonlein purpura nephritis. Kidney Int 80: 79-87, 2011.

3. Collard HR, Schwarz MI. Diffuse alveolar hemorrhage. Clin Chest Med 2004; 25:583.

4. Lai FM, Li EKM, Suen MWM, Lui SF, Li PKT, Lai KN. Pulmonary haemorrhage. A fatal manifestation in IgA nephropathy. Arch Pathol Lab Med 1994; 118: 542-546. 
5. Rajagopala S, Parameshwaran S, Ajmera JS, Ganesh RN, Katrevula A. Diffuse alveolar haemorrhage in $\operatorname{IgA}$ nephropathy: case series and systemic review of the literature. International Journal of rheumatic Diseases 2017:20: 109-121.

6. Anantham D, Chan KP, Chuah KL, Vathsala A, Eng P (2007) Pulmonary capillaritis in IgA nephropathy. South Med J 100, 605-7.

7. Rajagopala S, Shobha V, Devaraj U, D’Souza G, Garg I. Pulmonary hemorrhage in Henoch-Schonlein purpura: case report and systematic review of the English literature. Semin Arthritis Rheum 42: 391340, 2013. 